\title{
Drug treatment for chemotherapy-induced peripheral neuropathy in patients with pancreatic cancer
}

\author{
Mitsuru Sugimoto ${ }^{1)}$, Tadayuki Takagi ${ }^{1)}$, Rei Suzuki ${ }^{1)}$, Naoki Konno ${ }^{1)}$, Hiroyuki Asama ${ }^{1)}$, Yuki Sato ${ }^{1)}$, \\ Hiroki Irie $^{1)}$, Yoshinori Okubo ${ }^{122}$, Jun Nakamura ${ }^{122}$, Mika Takasumi ${ }^{1)}$, Minami Hashimoto ${ }^{122)}$, \\ Tsunetaka Kato ${ }^{122)}$, Ryoichiro Kobashi ${ }^{1)}$, Takuto Hikichi ${ }^{2)}$ and Hiromasa Ohira ${ }^{1)}$ \\ ${ }^{1)}$ Department of Gastroenterology, School of Medicine, Fukushima Medical University, Fukushima, Ja- \\ pan, ${ }^{2)}$ Department of Endoscopy, Fukushima Medical University Hospital, Fukushima, Japan
}

(Received November 9, 2021, accepted January 11, 2022)

\begin{abstract}
Pancreatic cancer (PC) is a lethal disease where most tumors are too advanced at diagnosis for resection, leaving chemotherapy as the mainstay of treatment. Although the prognosis of unresectable PC is poor, it has been dramatically improved by new chemotherapy treatments, such as the combination of 5-fluorouracil, oxaliplatin, irinotecan, and leucovorin (FOLFIRINOX) or gemcitabine plus nab-paclitaxel. However, as oxaliplatin and paclitaxel are common neurotoxic drugs, chemotherapy-induced peripheral neuropathy (CIPN) is a common and severe adverse effect of both treatments. As there are no agents recommended in the ASCO guidelines, we review the methods used to treat CIPN caused by PC treatment. The efficacy of duloxetine was observed in a large randomized controlled trial (RCT). In addition, pregabalin was more effective than duloxetine for CIPN in two RCTs. Although duloxetine and pregabalin can be effective for CIPN, they have several side effects. Therefore, the choice between the two drugs should be determined according to effect and tolerability. Mirogabalin is also used in patients with PC and there is hope it will yield positive outcomes when treating CIPN in the future.
\end{abstract}

Key words : Peripheral neuropathy, Chemotherapy, Pancreatic cancer, Oxaliplatin, Paclitaxel, Nabpaclitaxel

\section{Introduction}

Pancreatic cancer (PC) is a lethal disease. Worldwide, $\mathrm{PC}$ has become a major cause of cancerrelated death ${ }^{1-3)}$. One reason that the prognosis of $\mathrm{PC}$ is poor is that most cases are diagnosed after the tumor has reached an advanced stage and is thus difficult to resect ${ }^{4-6)}$. As a result, chemotherapy is the main treatment for PC patients. Although the prognosis for patients with $\mathrm{PC}$ patients is poor, it has been dramatically improved by new chemotherapy treatments, such as the combination of 5-fluorouracil, oxaliplatin, irinotecan, and leucovorin (FOLFIRI-
NOX) or gemcitabine plus nab-paclitaxel (GnP). In past reports, the median progression-free survival (PFS) was 3.0-20.4 months, and the median overall survival (OS) was 8.5-32.7 months with FOLFIRINOX therapy ${ }^{7,8)}$. The PFS was 4.4-8.4 months, and the median OS was 6.9-14.2 months with GnP therapy $^{9)}$. On the other hand, several adverse events of these effective therapies were reported. Chemotherapy-induced peripheral neuropathy (CIPN) is one of the common adverse events for both FOLFIRINOX therapy and GnP therapy. The frequency of grade $3-4$ CIPN has been reported to be $0-25 \%$ for FOLFIRINOX ${ }^{7,8,10,11)}$ and $1.8-30.4 \%$ for $\mathrm{GnP}$ ther-

Corresponding author: Mitsuru SugimotoＥ-mail : kita335@fmu.ac.jp

() 2022 The Fukushima Society of Medical Science. This article is licensed under a Creative Commons [Attribution-NonCommercial-ShareAlike 4.0 International] license.

https://creativecommons.org/licenses/by-nc-sa/4.0/ 
apy ${ }^{9)}$. Oxaliplatin and paclitaxel are common neurotoxic drugs ${ }^{12-14)}$. CIPN affects chemotherapy continuation. Therefore, adequate strategies for managing CIPN are needed. However, there are no agents recommended in the ASCO guidelines ${ }^{15)}$. The guidelines assembled randomized controlled trials (RCTs) or meta-analyses between January 2013 and February 2020. Although CIPN treatment is difficult, it should be used to continue chemotherapy. In this review, we studied strategies to manage peripheral sensory neuropathy that was induced by FOLFIRINOX and GnP (oxaliplatin and paclitaxel) by expanding the surveillance period and including other study formats.

\section{Materials and methods}

The literature was searched using the PubMed database from inception until November 2021. The combination of "chemotherapy-induced peripheral neuropathy" and each of the following keywords were used to search for CIPN treatment: "calcium and magnesium”, "goshajinkigan”, “duloxetine”, "vitamin B12”, "pregabalin”, "gabapentin”, and "pancreatic cancer". The reports that met the following terms participated in this review : (1) reports about CIPN treatment, (2) written in English, (3) CIPN induced by oxaliplatin or taxanes, and (4) only prospective studies. Moreover, we manually searched for additional reports that met the terms above or that were related to the subject of this review.

\section{Results}

A total of 347 reports were identified by using PubMed. Among them, 23 reports met the conditions written above and were included in this study.

\section{CIPN mechanism and symptoms}

CIPN is characterized by axonopathy, neuronopathy, and myelinopathy. Paclitaxel induces axonopathy. Taxanes prevent microtubule-based axonal transportation and have a harmful influence on downstream axonal biochemistry. Subsequently, the myelin sheath is impaired ${ }^{16)}$. Long axons are initially impaired ; therefore, patients often have sensory disorders that require them to wear compression gloves and/or stockings. Oxaliplatin induces neuronopathy. Combining DNA-modulating and platinum agents causes continuous damage to the neurons of the dorsal root ganglion, which accordingly causes apoptosis of dorsal root ganglion neurons ${ }^{17,18)}$. Axons and the myelin sheath are exponentially impaired. Both neurons with long axons and neurons with short axons are impaired, and sensory disorders are observed in the distal portions of the extremities or the body trunk.

\section{CIPN risk factors}

Menopause, elderly age, obesity, race, and alcohol consumption were identified as risk factors for CIPN that was induced by Taxol ${ }^{19-24)}$. In patients who were administered oxaliplatin, hypomagnesemia, hypoalbuminemia, anemia, and alcohol were identified as risk factors for $\mathrm{CIPN}^{25,26)}$.

\section{Diagnosis}

CIPN is generally diagnosed by clinical course. When a patient who is receiving neurotoxic antineoplastic agents experiences new pain or numbness in the distal portions of the extremities, the patient is diagnosed with CIPN.

In CIPN patients, disorders can be identified by neurological physical examination. CIPN patients show a lack of or lower action potentials and decreased conduction velocity of motor nerves or sensory nerves in studies of nerve conduction. However, these examinations are not often performed in everyday clinical practice $^{15)}$.

\section{Drug treatment}

In this section, prospective studies on drugs that are used to treat CIPN caused by oxaliplatin or taxanes are introduced. The results of these studies are summarized in Table $1 . \quad$ Calcium (Ca) and magnesium $(\mathrm{Mg})$ did not show efficacy in two double-blind RCTs ${ }^{27,28)}$. Goshajinkigan did not show efficacy in a large double-blind $\mathrm{RCT}^{29)}$. On the other hand, duloxetine showed efficacy in a large doubleblind $\mathrm{RCT}^{30)}$. Pregabalin did not show efficacy as a treatment for CIPN caused by oxaliplatin in a large double-blind $\mathrm{RCT}^{31)}$. However, pregabalin was more effective than duloxetine for CIPN in the two most recent double-blind $\mathrm{RCTs}^{32,33)}$. Gabapentin was ineffective for CIPN in a large double-blind $\mathrm{RCT}^{34)}$. The above results suggest that duloxetine is efficacious for treating CIPN. In addition, pregabalin has promising efficacy for treating CIPN (particularly for treating CIPN caused by taxanes).

Although duloxetine and pregabalin can be effective for CIPN, these drugs have several side effects, which are shown in Table $1^{32,35-37)}$. When the 
side effects that are shown in Table 1 become severe, discontinuing the drug and changing to another drug should be considered.

The details of the prospective studies are presented below.

\section{$C a$ and $M g$}

An elevated extracellular calcium concentration causes $\mathrm{N}+$ channels to close, which suppresses neuronal excitement ${ }^{38)}$. Two double-blind RCTs were performed to verify the efficacy of $\mathrm{Ca}$ and $\mathrm{Mg}$ for CIPN caused by oxaliplatin. In a study in 2010, Ishibashi et al. ${ }^{27)}$ divided 33 metastatic colon cancer patients into two groups. One group was administered $\mathrm{Ca} / \mathrm{Mg}$ before and after FOLFOX therapy. The other group received placebo. In that study, $\mathrm{Ca} / \mathrm{Mg}$ were not effective for $\mathrm{CIPN}(\mathrm{Ca} / \mathrm{Mg}$ group vs placebo : response rate was $36 \%$ vs $40 \%$, disease control rate was $73 \%$ vs $70 \%, p$ value $>0.99$ ). In a 2014 report, Loprinzi et al. ${ }^{28)}$ divided 353 postoperative patients into three groups. One group was administered $\mathrm{Ca} / \mathrm{Mg}$ before and after FOLFOX therapy $(n=118)$. Another group received placebo $(n=$ 119). The third group was administered $\mathrm{Ca} / \mathrm{Mg}$ only before FOLFOX therapy $(n=116)$. The time until Common Terminology Criteria for Adverse Events (CTCAE v4.0) grade 2 or higher CIPN was not significantly different between these three groups ( $\phi$ value $=0.3383$ ). In a 2018 prospective study written by Wesselink et al. ${ }^{39)}$, daily dietary intake of $\mathrm{Ca} / \mathrm{Mg}$ was evaluated in 196 colorectal cancer patients. In that study, daily dietary intake of $\mathrm{Mg}$ during chemotherapy was associated with a lower occurrence and severity of CIPN (occurrence ratio : $0.53,95 \%$ confidence interval $(\mathrm{CI})-1.95$, $-0.22)^{399}$.

\section{Goshajinkigan}

Four RCTs (three of which were double-blind) were performed to investigate the efficacy of goshajinkigan for treating CIPN caused by oxaliplatin. In two of the double-blind RCTs, goshajinkigan showed an obvious effect for treating CIPN ${ }^{40,41}$. However, goshajinkigan did not show efficacy in the largest double-blind RCT, as described above ${ }^{29)}$.

In a report written in 2010 , Nishioka $e t a l .{ }^{42)} \mathrm{di}-$ vided 45 metastatic or recurrent colon cancer patients who received chemotherapy involving oxaliplatin into a goshajinkigan group $(n=22)$ and a control group $(n=23)$. In the results, grade 3 CIPN (graded according to the neurotoxicity criteria of Debiopharm) was less frequent in the goshajinkigan group than in the control group (after 10 cours- es : $0 \%$ vs $12 \%$, after 20 courses : $33 \%$ vs $75 \%$, $\log$-rank test, $p$ value $<0.01)^{422}$. In a report written in 2012 (a double-blind RCT), Kaku et al. ${ }^{40)}$ divided ovarian cancer and uterine cancer patients who received paclitaxel plus carboplatin therapy into a group treated with vitamin B12 $(n=14)$ and a group treated with vitamin B12 plus goshajinkigan ( $n$ $=15)$. The incidence of abnormal current perception threshold value was significantly lower in the vitamin $\mathrm{B} 12+$ goshajinkigan group than in the vitamin B12 only group $(p \text { value }=0.024)^{40}$. In another report written in 2013 (a double-blind RCT), Kono et $a l .{ }^{41)}$ divided postoperative colon cancer patients who received FOLFOX therapy into a group treated with goshajinkigan $(n=44)$ and a group treated with placebo $(n=45)$. However, the frequency of grade 2 or higher CIPN (as graded by the CTCAE v3.0 criteria) was not significantly different between the two groups after 8 courses of chemotherapy (goshajinkigan : $39 \%$ vs placebo : $51 \%$, relative risk : 0.76 , 95\% CI 0.47-1.21).

In 2015, Oki et al. ${ }^{29)}$ reported a large doubleblind RCT. A total of 182 postoperative colon cancer patients were divided into a goshajinkigan group $(n=$ $89)$ and a placebo group $(n=93)$. The frequency of grade 2 or higher CIPN (as graded according to the CTCAE v3.0 criteria) was significantly greater in the group treated with goshajinkigan than in the group treated with placebo $(50.6 \% \text { vs } 31.2 \%)^{29}$. The internal use of goshajinkigan was significantly associated with the incidence of CIPN by a Cox proportional hazards analysis (hazard ratio : $1.908, p$ value $=$ 0.007).

\section{Duloxetine}

Norepinephrine and serotonin prevent peripheral pain stimulation from reaching dorsal horn neurons $^{43)}$. Duloxetine mitigates neuropathy by inhibiting the reuptake of norepinephrine and serotonin ${ }^{44)}$.

Three RCTs investigated the efficacy of duloxetine for treating CIPN. In a report written in 2013 (a double-blind RCT), Smith et al. ${ }^{30}$ ) divided cancer patients with CIPN who were administered Taxol or platinum-based drugs into a group $(n=115)$ treated with duloxetine (30 $\mathrm{mg}$ a day for a week; then 60 $\mathrm{mg}$ a day for four weeks) and a group treated with placebo $(n=116)$. CIPN was evaluated by the $\mathrm{Nu}-$ merical Rating Scale (NRS). In the group treated with duloxetine, CIPN was significantly improved (mean decrease on average pain: duloxetine group $1.06,95 \%$ CI $0.72-1.40$ vs placebo group $0.34,95 \%$ CI $0.01-0.66, p$ value $=0.003)^{30)}$. In another report written by Hirayama et al. ${ }^{35)}, 34$ CIPN participants 


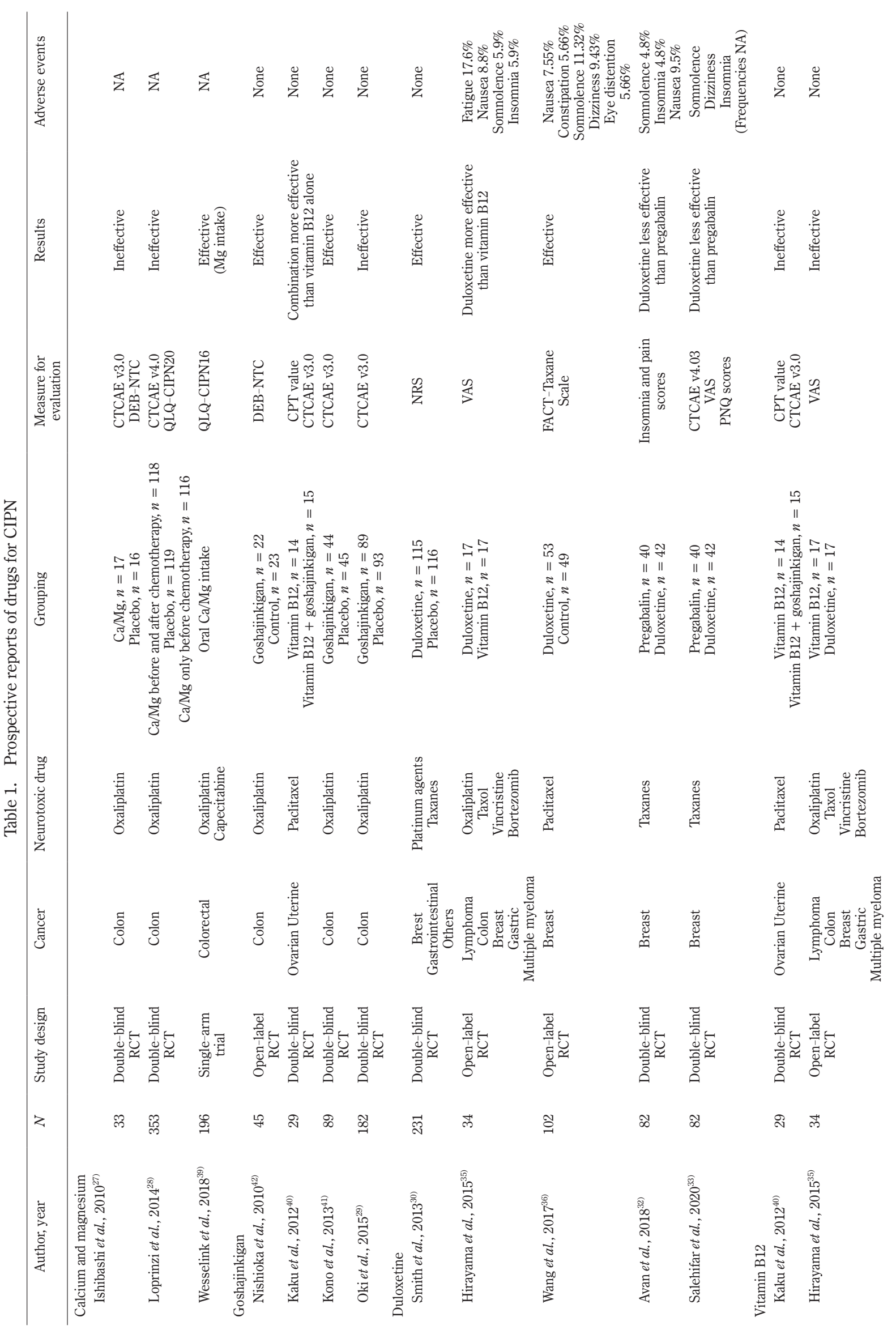




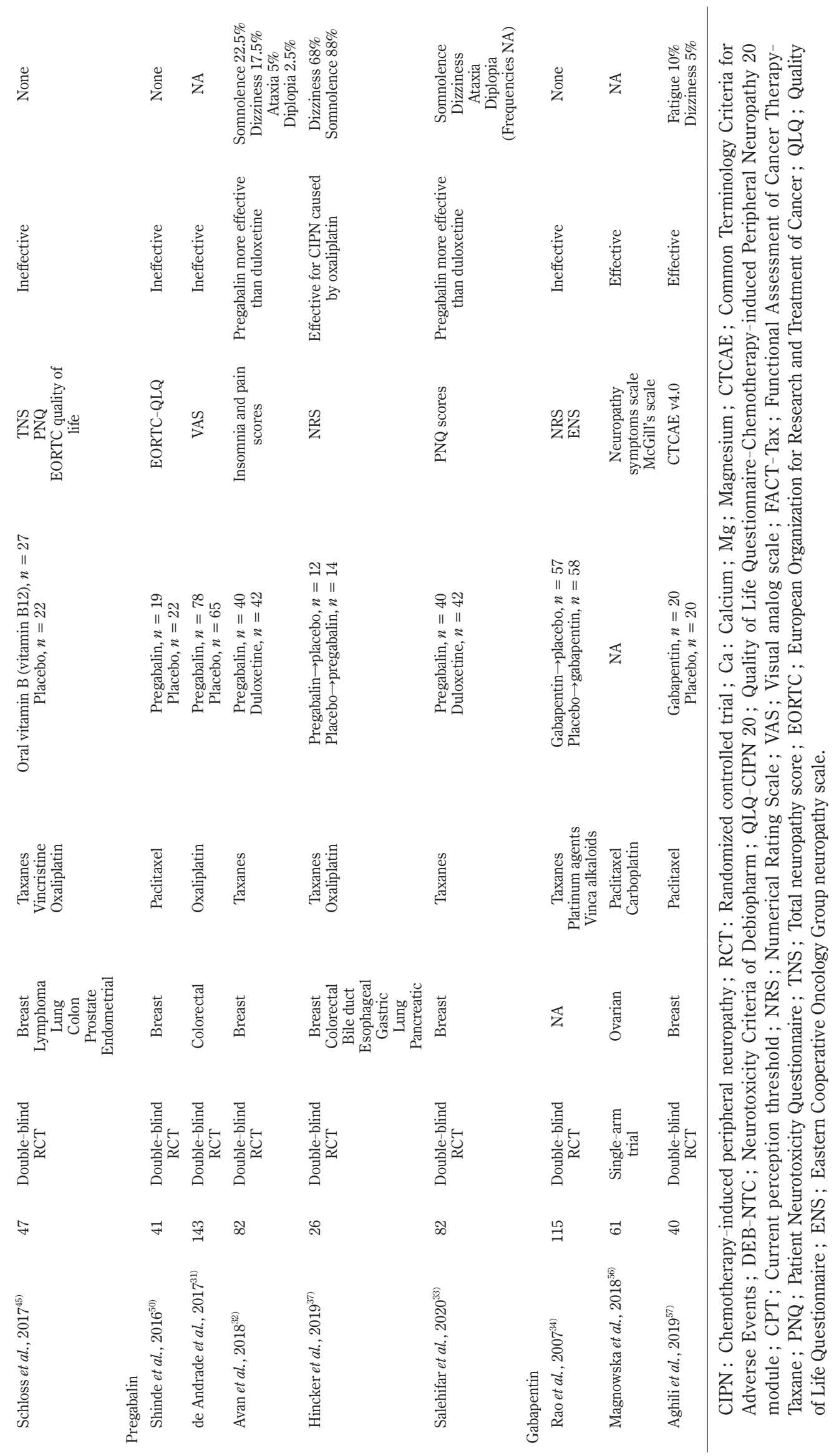


were administered paclitaxel, oxaliplatin, vincristine, or bortezomib. The patients were divided into a duloxetine group $(n=17)$ (20 $\mathrm{mg}$ a day for a week ; then $40 \mathrm{mg}$ a day for three weeks) and a vitamin B12 group $(n=17)$. Pain and numbness were evaluated by the visual analog scale (VAS) and were significantly improved in the duloxetine group compared with the vitamin B12 group (the mean differences in VAS scores between pretreatment and after four weeks treatment : numbness, $p$ value $=$ 0.03 ; pain, $p=0.04)^{35)}$. In a RCT reported by Wang et al. ${ }^{36)}$ in 2017, 102 breast cancer patients underwent treatment for CIPN. They were classified into a duloxetine group $(n=53)$ (30 $\mathrm{mg}$ a day for 4 weeks ; then $60 \mathrm{mg}$ a day for 8 weeks) and a control group ( $n=49$ ), which was treated with NSAIDs, fish oil, and vitamin B. The Functional Assessment of Cancer Therapy - Taxane Scale result was significantly decreased in the duloxetine group compared with the control group (the median $\left(25^{\text {th }}-75^{\text {th }}\right.$ percentile) decreasing difference was $4(2-6)$ vs $1(0-4), p$ value $=0.005)^{36)}$.

In addition, duloxetine has been used as the control agent in two double-blind RCTs studying pregabalin $^{32,33)}$. In these studies, duloxetine was less effective than pregabalin for treating CIPN. The details of these studies are provided in the pregabalin section.

Some adverse events were reported in the above RCTs (fatigue $17.6 \%$, nausea $7.55-9.5 \%$, somnolence $4.8-11.32 \%$, insomnia $4.8-5.9 \%$, constipation $5.66 \%$, dizziness $9.43 \%$, and eye distention $5.66 \%)^{32,35,36)}$.

\section{Vitamin B12}

Vitamin B12 was used as a control treatment in two RCTs studying goshajinkigan and duloxetine $^{35,40)}$. In these studies, vitamin B12 did not show better efficacy than goshajinkigan or duloxetine for relieving CIPN. In 2017, Schloss et al. ${ }^{45)}$ reported a double-blind RCT. Sixty-nine patients were divided into a vitamin B group, in which patients received oral vitamin B (vitamin B12), and a placebo group. The vitamin B group did not show better relief of CIPN than the placebo group (the reduction in total neuropathy score after 12,24 , and 36 weeks treatment : $p$ value $=0.73)^{45)}$.

\section{Pregabalin}

Pregabalin connects the $\alpha 2 \delta$ subunit of the $\mathrm{Ca}$ channel in the back horn of the spinal cord and prevents the inflow of calcium that is necessary for releasing neurotransmitters ${ }^{46-49)}$.

Five RCTs were performed to investigate the efficacy of pregabalin for treating CIPN. In 2016, Shinde et $a l .^{50)}$ reported a multicenter double-blind RCT. Overall, 41 patients who received weekly paclitaxel therapy were divided into a pregabalin $(n=$ $19,150 \mathrm{mg}$ a day for 12 weeks) group and a placebo group $(n=22)$. In the study, CIPN was not relieved by pregabalin (the European Organization for Research and Treatment of Cancer Quality of Life (EORTC-QLQ) CIPN20 questionnaire, mean (standard deviation) : worse pain, pregabalin : 2.6 (2.5) vs placebo : $3.2(3.0), p$ value $=0.56$; average pain, pregabalin : $2.6(2.2)$ vs placebo : $2.2(2.6), p=$ $0.48)^{50)}$. In 2017, de Andrade et al. ${ }^{31)}$ performed a double-blind RCT. A total of 143 colorectal cancer patients who received oxaliplatin were divided into a pregabalin (150-600 mg a day) group $(n=78)$ and a placebo group $(n=65)$. Patients received pregabalin or placebo for three days before and three days after each infusion of oxaliplatin. However, pregabalin efficacy was not observed (Quality of life score : pregabalin : $79.4 \pm 20.6$ vs placebo : $76.9 \pm 23.1)^{31)}$. In 2018, Avan et $a .^{32)}$ performed another doubleblind RCT. Eighty-two breast cancer patients with taxane-induced sensory neuropathy were divided into a pregabalin group ( $n=40,75 \mathrm{mg}$ a day for a week ; then $75 \mathrm{mg}$ twice a day for five weeks) and a duloxetine group ( $n=42,30 \mathrm{mg}$ a day for a week ; then $30 \mathrm{mg}$ twice a day for five weeks). CIPN pain was more relieved by pregabalin than by duloxetine, and pregabalin was associated with greater improvement in insomnia and pain scores of EORTC-QLQ C30 $(p \text { value }=0.05, p \text { value }<0.001)^{32}$. In 2019, Hincker et $a l .{ }^{37)}$ performed another double-blind RCT. Twenty-six patients with CIPN treated with oxaliplatin, docetaxel, and paclitaxel were divided into a pregabalin then placebo group ( $n=12$, maximum $600 \mathrm{mg}$ a day for four weeks) and a placebo then pregabalin group $(n=14)$. In a study, CIPN caused by oxaliplatin was more improved by pregabalin than by placebo (reduction in pain : pregabalin : $35.4 \%$ vs placebo : $14.6 \%, p$ value $=0.04)^{37)}$. In 2020, Salehifar et al. ${ }^{33)}$ reported another double-blind RCT. Eighty-two breast cancer patients with grade 1 or greater neuropathy (as assessed by the CTCAE v4.03 criteria) caused by paclitaxel or docetaxel were divided into a pregabalin group $(n=40,150$ $\mathrm{mg}$ a day) and a duloxetine group ( $n=42,60 \mathrm{mg}$ a day). The Patient Neurotoxicity Questionnaire (PNQ) scores in the pregabalin group were better than those in the duloxetine group (improving the PNQ scores : pregabalin : $90 \%$ vs duloxetine : $31 \%, p$ value $<0.001)^{33)}$

Some adverse events were reported in the 
above RCTs (somnolence 22.5-88\%, dizziness 17.5$68 \%$, ataxia $5.0 \%$, and diplopia $2.5 \%)^{32,37)}$.

\section{Gabapentin}

The mechanism by which gabapentin alleviates CIPN is identical to that of pregabalin. Gabapentin inhibits the $\alpha 2 \delta 1$ subunit of $\mathrm{Ca}$ channels and prevents the release of neurotransmitters ${ }^{46,47,51-55)}$.

Three prospective studies have shown the efficacy of gabapentin for treating CIPN. In 2007, Rao et $a{ }^{34)}$ performed a double-blind RCT that targeted 115 patients who had experienced CIPN induced by taxanes, platinum agents, or vinca alkaloids for a month or longer. The drug dosing methods were as follows : patients took gabapentin or placebo for six weeks and then crossed over two weeks after drug withdrawal. Gabapentin was initiated at 300 $\mathrm{mg}$ a day and was gradually increased to 2,700 mg. The symptoms caused by CIPN were not improved by gabapentin (average pain score of NRS, before crossover : gabapentin : 3.3 vs placebo : 3.1 , $p$ value $=0.8$, after crossover $:$ gabapentin $: 2.5 \mathrm{vs}$ placebo : $3.1, p$ value $=0.2)^{34)}$. In 2018, Magnowska et $a l .{ }^{56)}$ reported a prospective study. Sixty-one ovarian cancer patients who received paclitaxel and carboplatin chemotherapy (6 courses every 3 weeks) were enrolled. CIPN occurred in $78.7 \%$ of the patients. CIPN was improved in patients who took gabapentin (Neuropathy symptoms scale : pretreatment vs a month after treatment, $p$ value $=0.027)^{56)}$. In 2019, Aghili et al. ${ }^{57)}$ reported a double-blind RCT. Forty breast cancer patients who received paclitaxel chemotherapy were divided into a gabapentin group ( $n=20,300 \mathrm{mg}$ three times a day for 2 weeks starting at day 1 of each cycle) and a placebo group ( $n=$ 20). The grade of CIPN was evaluated according to the CTCAE v4.0 criteria. The frequency of second- and third-grade CIPN was significantly lower in the gabapentin group than in the placebo group (grades 2 and 3 CIPN after 4 cycle chemotherapy : gabapentin : $1 \%$ vs placebo : $90 \%, p$ value $=$ $0.000)^{57)}$.

\section{CIPN treatment and pancreatic cancer}

As described above, CIPN occurs in FOLFIRINOX and GnP therapy. However, few reports have addressed CIPN in a sufficient number of PC patients. In previous reports, CIPN was not statistically associated with poor prognoses of unresectable $\mathrm{PC}$ patients. Overall survival was longer in patients with CIPN than in patients without CIPN. In 2018, You et al. ${ }^{58)}$ reported that OS was longer in metastatic PC patients with neuropathy $(n=52$,
15.53 months) than in metastatic PC patients without neuropathy $(n=36,10.13$ months, $P$ value $=$ 0.007). In 2021, Catalano et $a l .{ }^{59)}$ reported that the median OS was 13 months and 10 months in metastatic PC patients with $(n=47)$ and without grade $1-2$ CIPN $(n=106)(P$ value $=0.04)$. In the multivariate analysis, grade 1-2 neuropathy was independently associated with OS (HR 0.65 ; 95\% CI 0.45$0.98 ; P$ value $=0.03$ ). The reason OS was longer in PC patients with CIPN is unknown. Patients with longer OS are administered more anticancer drugs ; therefore, the OS of PC patients with CIPN might be longer than the OS of $\mathrm{PC}$ patients without CIPN. However, some PC patients with CIPN discontinued chemotherapy ${ }^{58,60)}$. Therefore, CIPN should be treated by the treatment described above.

Recently, the use of mirogabalin for CIPN was covered by insurance in Japan. Previously, mirogabalin was used for diabetes-related peripheral neuropathy ${ }^{61)}$. We have reported on the efficacy of mirogabalin for CIPN in PC patients ${ }^{62)}$. In the study, $163 \mathrm{PC}$ patients received FOLFIRINOX or $\mathrm{GnP}$ therapy. Among them, 34 patients experienced CIPN that was grade 2 or higher according to the CTCAE ver 5.0. Thirteen patients were treated with mirogabalin, and 21 patients were treated with pregabalin. The improvement of CIPN at 2, 4, and 6 weeks after treatment was more apparent in the mirogabalin group than in the pregabalin group (2 weeks : $84.6 \%(11 / 13)$ vs $33.3 \%(7 / 21), p$ value $=$ $0.005 ; 4$ weeks, and 6 weeks : $92.3 \%(12 / 13)$ vs $33.3 \%(7 / 21), p$ value $=0.001)$. Though the result was retrospective, future development of mirogabalin treatment for CIPN is expected.

\section{Conclusion}

Oxaliplatin and nab-paclitaxel are neurotoxic drugs used in chemotherapy for PC. There is no statistical evidence that CIPN is associated with poor prognoses for patients with PC. However, CIPN can be so serious that chemotherapy is discontinued. There are few reports that addresses drug treatment for CIPN in PC patients. Duloxetine and pregabalin are reported to be effective in taxanes and oxaliplatin-induced neuropathy in patients with several cancers. Some side effects were observed by using duloxetine and pregabalin. Drugs should be carefully selected according to tolerance. Additionally, mirogabalin is currently used for the treatment of CIPN, and the continued efficacy of mirogabalin is expected in the future. 


\section{Acknowledgements}

We thank all the staff at the Department of Gastroenterology of Fukushima Medical University, the Department of Endoscopy of Fukushima Medical University Hospital, and the gastroenterology ward of Fukushima Medical University Hospital. We also thank American Journal Experts for providing English language editing.

\section{Conflict of interest disclosure}

The authors have no conflicts of interest to declare.

\section{References}

1. Bray F, Ferlay J, Soerjomataram I, Siegel RL, Torre LA, Jemal A. Global cancer statistics 2018 : GLOBOCAN estimates of incidence and mortality worldwide for 36 cancers in 185 countries. CA Cancer J Clin, 68 : 394-424, 2018.

2. Siegel RL, Miller KD, Jemal A. Cancer statistics, 2020. CA Cancer J Clin, 70 : 7-30, 2020.

3. Afshar N, English DR, Chamberlain JA, et al. Differences in cancer survival by remoteness of residence : an analysis of data from a populationbased cancer registry. Cancer Causes Control, $31:$ : 617-629, 2020.

4. Stathis A, Moore MJ. Advanced pancreatic carcinoma : current treatment and future challenges. Nat Rev Clin Oncol, 7 : 163-172, 2010.

5. Warsame R, Grothey A. Treatment options for advanced pancreatic cancer : a review. Expert Rev Anticancer Ther, 12 : 1327-1336, 2012.

6. Fogel EL, Shahda S, Sandrasegaran K, et al. A Multidisciplinary Approach to Pancreas Cancer in 2016: A Review. Am J Gastroenterol, 112 : 537-554, 2017.

7. Suker M, Beumer BR, Sadot E, et al. FOLFIRINOX for locally advanced pancreatic cancer : a systematic review and patient-level meta-analysis. Lancet Oncol, 17 : 801-810, 2016.

8. Thibodeau S, Voutsadakis IA. FOLFIRINOX chemotherapy in metastatic pancreatic cancer : a systematic review and meta-analysis of retrospective and phase II studies. J Clin Med, 7 : 7, 2018.

9. Zhang Y, Xu J, Hua J, et al. Nab-paclitaxel plus gemcitabine as first-line treatment for advanced pancreatic cancer : a systematic review and metaanalysis. J Cancer, 10 : 4420-4429, 2019.

10. Lorgis V, Chauffert B, Gentil J, Ghiringhelli F. Influcence of localization of primary tumor on effectiveness of 5-fluorouracil/leucovorin combined with irinotecan and oxaliplatin (FOLFIRINOX) in patients with metastatic pancreatic adenocarcinoma : a retrospective study. Anticancer Res, 32 : 4125-4130, 2012.

11. Tong H, Fan Z, Liu B, Lu T. The benefits of modified FOLFIRINOX for advanced pancreatic cancer and its induced adverse events : a systematic review and meta-analysis. Sci Rep, 8 : 8666, 2018.

12. Loprinzi CL, Reeves BN, Dakhil SR, et al. Natural history of paclitaxel-associated acute pain syndrome : prospective cohort study NCCTG N08C1. J Clin Oncol, 29 : 1472-1478, 2011.

13. Reeves BN, Dakhil SR, Sloan JA, et al. Further data supporting that paclitaxel-associated acute pain syndrome is associated with development of peripheral neuropathy : North Central Cancer Treatment Group trial N08C1. Cancer, 118 : 5171-5178, 2012.

14. Pachman DR, Qin R, Seisler DK, et al. Clinical Course of Oxaliplatin-Induced Neuropathy : Results From the Randomized Phase III Trial N08CB (Alliance). J Clin Oncol, 33 : 3416-3422, 2015.

15. Loprinzi CL, Lacchetti C, Bleeker J, et al. Prevention and management of chemotherapy-induced peripheral neuropathy in survivors of adult cancers : ASCO guideline update. J Clin Oncol, 38 : 3325-3348, 2020.

16. Morfini GA, Burns M, Binder LI, et al. Axonal transport defects in neurodegenerative diseases. J Neurosci, 29 : 12776-12786, 2009.

17. Gill JS, Windebank AJ. Cisplatin-induced apoptosis in rat dorsal root ganglion neurons is associated with attempted entry into the cell cycle. J Clin Invest, 101 : 2842-2850, 1998.

18. McDonald ES, Windebank AJ. Cisplatin-induced apoptosis of DRG neurons involves bax redistribution and cytochrome c release but not fas receptor signaling. Neurobiol Dis, 9 : 220-233, 2002.

19. Rowinsky EK, Chaudhry V, Cornblath DR, Donehower RC. Neurotoxicity of taxol. J Natl Cancer Inst Monogr, 107-115, 1993.

20. Rowinsky EK, Eisenhauer EA, Chaudhry V, Arbuck SG, Donehower RC. Clinical toxicities encountered with paclitaxel (Taxol). Semin Oncol, 20 : 1-15, 1993.

21. Gogas H, Shapiro F, Aghajanian C, et al. The impact of diabetes mellitus on the toxicity of therapy for advanced ovarian cancer. Gynecol Oncol, 61 : 22-26, 1996.

22. Schneider BP, Zhao F, Wang M, et al. Neuropathy is not associated with clinical outcomes in patients receiving adjuvant taxane-containing therapy for operable breast cancer. J Clin Oncol, 30 : 30513057, 2012.

23. Schneider BP, Li L, Radovich M, et al. GenomeWide Association Studies for Taxane-Induced Pe- 
ripheral Neuropathy in ECOG-5103 and ECOG1199. Clin Cancer Res, 21 : 5082-5091, 2015.

24. Bao T, Basal C, Seluzicki C, Li SQ, Seidman AD, Mao JJ. Long-term chemotherapy-induced peripheral neuropathy among breast cancer survivors : prevalence, risk factors, and fall risk. Breast Cancer Res Treat, 159 : 327-333, 2016.

25. Smith EM, Beck SL, Cohen J. The total neuropathy score : a tool for measuring chemotherapy-induced peripheral neuropathy. Oncol Nurs Forum, 35 : 96-102, 2008.

26. Stubblefield MD, McNeely ML, Alfano CM, Mayer DK. A prospective surveillance model for physical rehabilitation of women with breast cancer : chemotherapy-induced peripheral neuropathy. Cancer, 118 : 2250-2260, 2012.

27. Ishibashi K, Okada N, Miyazaki T, Sano M, Ishida H. Effect of calcium and magnesium on neurotoxicity and blood platinum concentrations in patients receiving $\mathrm{mFOLFOX6}$ therapy : a prospective randomized study. Int J Clin Oncol, 15 : 82-87, 2010.

28. Loprinzi CL, Qin R, Dakhil SR, et al. Phase III randomized, placebo-controlled, double-blind study of intravenous calcium and magnesium to prevent oxaliplatin-induced sensory neurotoxicity (N08CB/ Alliance). J Clin Oncol, 32 : 997-1005, 2014.

29. Oki E, Emi Y, Kojima H, et al. Preventive effect of Goshajinkigan on peripheral neurotoxicity of FOLFOX therapy (GENIUS trial) : a placebo-controlled, double-blind, randomized phase III study. Int J Clin Oncol, 20 : 767-775, 2015.

30. Smith EM, Pang H, Cirrincione C, et al. Effect of duloxetine on pain, function, and quality of life among patients with chemotherapy-induced painful peripheral neuropathy : a randomized clinical trial. JAMA, 309 : 1359-1367, 2013.

31. de Andrade DC, Jacobsen Teixeira M, Galhardoni R, et al. Pregabalin for the Prevention of OxaliplatinInduced Painful Neuropathy : A Randomized, Double-Blind Trial. Oncologist, 22 : 1154-e1105, 2017.

32. Avan R, Janbabaei G, Hendouei N, et al. The effect of pregabalin and duloxetine treatment on quality of life of breast cancer patients with taxaneinduced sensory neuropathy : a randomized clinical trial. J Res Med Sci, 23 : 52, 2018.

33. Salehifar E, Janbabaei G, Hendouei N, Alipour A, Tabrizi N, Avan R. Comparison of the efficacy and safety of pregabalin and duloxetine in taxane-induced sensory neuropathy : a randomized controlled trial. Clin Drug Investig, 40 : 249-257, 2020.

34. Rao RD, Michalak JC, Sloan JA, et al. Efficacy of gabapentin in the management of chemotherapyinduced peripheral neuropathy : a phase 3 randomized, double-blind, placebo-controlled, crossover trial (N00C3). Cancer, 110 : 2110-2118, 2007.

35. Hirayama Y, Ishitani K, Sato Y, et al. Effect of duloxetine in Japanese patients with chemotherapyinduced peripheral neuropathy : a pilot randomized trial. Int J Clin Oncol, 20 : 866-871, 2015.

36. Wang J, Li Q, Xu B, Zhang T, Chen S, Luo Y. Efficacy and safety of duloxetine in Chinese breast cancer patients with paclitaxel-induced peripheral neuropathy. Chin J Cancer Res, 29 : 411-418, 2017.

37. Hincker A, Frey K, Rao L, et al. Somatosensory predictors of response to pregabalin in painful chemotherapy-induced peripheral neuropathy : a randomized, placebo-controlled, crossover study. Pain, 160 : 1835-1846, 2019.

38. Armstrong CM, Cota G. Calcium block of $\mathrm{Na}+$ channels and its effect on closing rate. Proc Natl Acad Sci U S A, 96 : 4154-4157, 1999.

39. Wesselink E, Winkels RM, van Baar H, et al. Dietary intake of magnesium or calcium and chemotherapy-induced peripheral neuropathy in colorectal cancer patients. Nutrients, 10 : 398, 2018.

40. Kaku H, Kumagai S, Onoue H, et al. Objective evaluation of the alleviating effects of Goshajinkigan on peripheral neuropathy induced by paclitaxel/carboplatin therapy : A multicenter collaborative study. Exp Ther Med, 3 : 60-65, 2012.

41. Kono T, Hata T, Morita S, et al. Goshajinkigan oxaliplatin neurotoxicity evaluation (GONE) : a phase 2, multicenter, randomized, doubleblind, placebocontrolled trial of Goshajinkigan to prevent oxaliplatininduced neuropathy. Cancer Chemother Pharmacol, 72 : 1283-1290, 2013.

42. Nishioka M, Shimada M, Kurita N, et al. The Kampo medicine, Goshajinkigan, prevents neuropathy in patients treated by FOLFOX regimen. Int J Clin Oncol, 16 : 322-327, 2011.

43. Willis WD, Westlund KN. Neuroanatomy of the pain system and of the pathways that modulate pain. J Clin Neurophysiol, 14 : 2-31, 1997.

44. Hershman DL, Lacchetti C, Dworkin RH, et al. Prevention and management of chemotherapy-induced peripheral neuropathy in survivors of adult cancers : American society of clinical oncology clinical practice guideline. J Clin Oncol, 32 : 1941-1967, 2014.

45. Schloss JM, Colosimo M, Airey C, Masci P, Linnane AW, Vitetta L. A randomised, placebo-controlled trial assessing the efficacy of an oral B group vitamin in preventing the development of chemotherapy-induced peripheral neuropathy (CIPN). Support Care Cancer, 25 : 195-204, 2017.

46. Xiao W, Boroujerdi A, Bennett GJ, Luo ZD. Chemotherapy-evoked painful peripheral neuropathy : analgesic effects of gabapentin and effects on expression of the alpha-2-delta type-1 calcium 
channel subunit. Neuroscience, $144: 714-720$, 2007.

47. Gauchan P, Andoh T, Ikeda K, et al. Mechanical allodynia induced by paclitaxel, oxaliplatin and vincristine : different effectiveness of gabapentin and different expression of voltage-dependent calcium channel alpha(2)delta-1 subunit. Biol Pharm Bull, 32 : 732-734, 2009.

48. Peng P, Xi Q, Xia S, et al. Pregabalin attenuates docetaxel-induced neuropathy in rats. J Huazhong Univ Sci Technolog Med Sci, 32 : 586-590, 2012.

49. Aoki M, Kurauchi Y, Mori A, Nakahara T, Sakamoto $\mathrm{K}$, Ishii K. Comparison of the effects of single doses of elcatonin and pregabalin on oxaliplatin-induced cold and mechanical allodynia in rats. Biol Pharm Bull, 37 : 322-326, 2014.

50. Shinde SS, Seisler D, Soori G, et al. Can pregabalin prevent paclitaxel-associated neuropathy? An ACCRU pilot trial. Support Care Cancer, 24 : 547-553, 2016.

51. Field MJ, Oles RJ, Lewis AS, McCleary S, Hughes J, Singh L. Gabapentin (neurontin) and S-(+)3 -isobutylgaba represent a novel class of selective antihyperalgesic agents. $\mathrm{Br} \mathrm{J}$ Pharmacol, 121 : 1513-1522, 1997.

52. Shimoyama M, Shimoyama N, Hori Y. Gabapentin affects glutamatergic excitatory neurotransmission in the rat dorsal horn. Pain, 85 : 405-414, 2000.

53. Luo ZD, Chaplan SR, Higuera ES, et al. Upregulation of dorsal root ganglion (alpha)2(delta) calcium channel subunit and its correlation with allodynia in spinal nerve-injured rats. J Neurosci, 21 : 1868-1875, 2001.

54. Lynch JJ, 3rd, Wade CL, Zhong CM, Mikusa JP, Honore P. Attenuation of mechanical allodynia by clinically utilized drugs in a rat chemotherapy-induced neuropathic pain model. Pain, 110 : 56-63, 2004 .
55. Ling B, Authier N, Balayssac D, Eschalier A, Coudore F. Behavioral and pharmacological description of oxaliplatin-induced painful neuropathy in rat. Pain, 128 : 225-234, 2007.

56. Magnowska M, Iżycka N, Kapoła-Czyż J, et al. Effectiveness of gabapentin pharmacotherapy in chemotherapy-induced peripheral neuropathy. Ginekol Pol, 89 : 200-204, 2018.

57. Aghili M, Zare M, Mousavi N, et al. Efficacy of gabapentin for the prevention of paclitaxel induced peripheral neuropathy: A randomized placebo controlled clinical trial. Breast J, 25 : 226-231, 2019.

58. You MS, Ryu JK, Choi YH, et al. Efficacy of Nabpaclitaxel plus gemcitabine and prognostic value of peripheral neuropathy in patients with metastatic pancreatic cancer. Gut Liver, 12 : 728-735, 2018.

59. Catalano M, Aprile G, Ramello M, Conca R, Petrioli R, Roviello G. Association between low-grade chemotherapy-induced peripheral neuropathy (CINP) and survival in patients with metastatic adenocarcinoma of the pancreas. J Clin Med, 10 : 1846, 2021.

60. Von Hoff DD, Ervin T, Arena FP, et al. Increased survival in pancreatic cancer with nab-paclitaxel plus gemcitabine. N Engl J Med, 369 : 16911703, 2013.

61. Baba M, Matsui N, Kuroha M, Wasaki Y, Ohwada S. Mirogabalin for the treatment of diabetic peripheral neuropathic pain : A randomized, doubleblind, placebo-controlled phase III study in Asian patients. J Diabetes Investig, 10 : 1299-1306, 2019.

62. Sugimoto M, Takagi T, Suzuki R, et al. Mirogabalin vs pregabalin for chemotherapy-induced peripheral neuropathy in pancreatic cancer patients. BMC Cancer, 21 : 1319, 2021. 